\title{
The experimenter module of the DSMAC (Dynamic Sequential MultiAttribute Choice) interface
}

\author{
GAD SAAD \\ Concordia University, Montreal, Canada
}

\begin{abstract}
The DSMAC (Dynamic Sequential MultiAttribute Choice) interface is a process-tracing methodology that permits a researcher to investigate subjects' behaviors in an optional-stopping task. In such an environment, an individual decides after each piece of acquired information whether to make a choice between a pair of competing alternatives or to acquire additional information. DSMAC consists of two modules - an experimenter and a subject interface. The former allows the researcher to specify all of the relevant experimental parameters for a particular study, which is subsequently used as input by the latter. The bimodular nature of DSMAC significantly reduces the set-up time required for constructing novel stimuli for new experiments. DSMAC runs on a Macintosh computer. It is compatible with most Mactintosh models that use a system 7.0 (or higher) operating system. The source code was written in ThinkPascal 4.0.1.
\end{abstract}

Saad (1996) described the SMAC interface, a computerized data collection tool for investigating Sequential MultiAttribute Choices. Such a process assumes that a decision maker iteratively acquires one piece of attribute information at a time, across all competing alternatives, until sufficient discrimination is achieved to permit a final choice. Within SMAC, three distinct stages of the sequential process can be investigated: (1) the attribute selection stage - namely, deciding which attribute to acquire next, if additional information is desired; (2) the information integration stage, that is, the processing of newly acquired information; and (3) the backtracking stage, that is, the review of previously acquired information.

The sequential-sampling approach was first proposed by Wald (1947) in his development of statistical decision theory. Unlike classical statistical theory, in which the sample size used to test a hypothesis is fixed a priori, Wald's iterative approach argued that the sample size itself could be a random variable. In other words, after each newly acquired observation, one decides whether to reject/ not reject the hypothesis or to sample additional information. Within the multiattribute decision-making literature, the sequential-sampling paradigm has been uti-

The author thanks Salim Samaha and Darren Bicknell for their research assistanceships. The former programmed the interface, whereas much of the crash testing and debugging responsibilities were performed by the latter. In addition, the author is grateful to the Social Sciences and Humanities Research Council of Canada for its financial support. Correspondence should be addressed to G. Saad, Concordia University, 1455 de Maisonneuve Blvd. W., Montreal, PQ, Canada H3G IM8 (e-mail: gadsaad@vax2.concordia.ca). lized by several researchers (see Aschenbrenner, Albert, \& Schmalhofer, 1984; Busemeyer \& Townsend, 1993; Diederich, 1995; Saad \& Russo, 1996).

SMAC performed only one experimental task. Modifications to the experimental task required source code alterations. The current Dynamic SMAC (DSMAC) interface was developed in order to alleviate the static nature of the original SMAC interface. It consists of two modules - an experimenter and a subject interface. The former is an interactive shell that allows an experimenter to explicitly specify all of the parameters of a particular study. Subsequently, the subject interface uses the parameters as input, in tailor-making the task environment that the subjects will be exposed to. The purpose of the current paper is to present all of the intricate details of the experimenter module of DSMAC. For an overview of the subject interface, the reader is referred to Saad (1996).

\section{EXPERIMENTER MODULE}

1. DSMAC allows the researcher to define both the experimental stimulus (e.g., choice between graduate schools to attend) and the corresponding attributes defining it (e.g., rank of the university, tuition, beauty of campus, student/faculty ratio, and quality of student life). Similarly, one can specify the experimental task, including the number of attributes that define the stimulus and the number of binary choices that the subjects will make. Both of the latter parameters can take on any value from 1 through 25.

2. DSMAC permits the researcher to specify which attributes (if any) will carry directionality and their corre- 


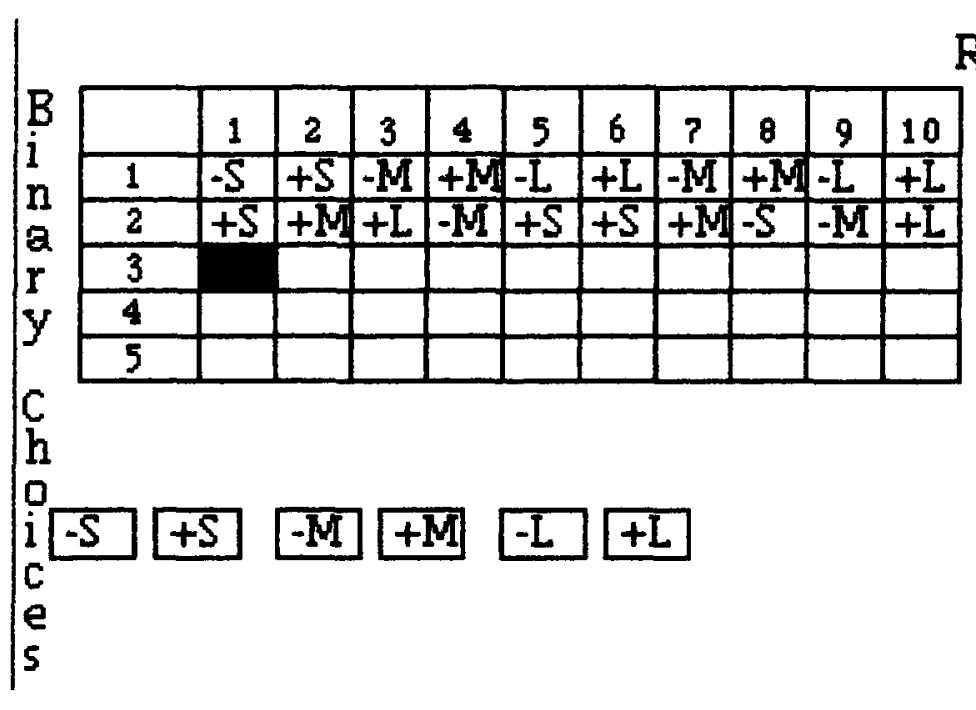

Rank of Attributes

Figure 1. The trial configuration table specifies the magnitude of the attribute differences (small, medium, or large) and whom they will favor $(+/-)$. The first row corresponds to a difficult choice yielding little differentiation between the two competing alternatives, whereas the second now would yield highly differentiated alternatives. The dimensions of the table (i.e., $5 \times 10$ ) imply that the subject will make five binary choices and that he or she can acquire up to 10 attributes prior to making a final choice between a pair of competing alternatives.

sponding direction. For example, if the experimental task involves choosing between pairs of apartments, neighborhood safety might carry directionality, since, ceteris paribus, safe neighborhoods should always be preferred to dangerous ones. In yet other circumstances, one might not wish to and/or be unable to define a specific directionality (e.g., rent).

3. The researcher can specify the attribute-listing format in both the attribute selection and backtracking screens. Three options are available-namely, alphabetical, rank ordered (subject specific), or random. For the latter format, the randomization occurs at the start of each new binary choice.

4. Recall that backtracking refers to the review of previously acquired information. Passive backtracking implies that one can review information without the possibility of modifying its previously entered impact, whereas online backtracking permits such modifications. In DSMAC, a researcher can specify whether the subject module should support online backtracking or simply its passive counterpart. For example, assume that, on seeing that universities A and B were ranked $3 \mathrm{rd}$ and 10 th respectively, a subject entered an increase of $10 \%$ in cumulative confidence favoring university A. Online backtracking would allow the subject not only to review the latter information but also to modify, if so desired, the previously entered change of $10 \%$.

5 . The researcher can specify whether the attribute ranking/weighing procedure will take place solely at the start of the experiment or at both the start and the end of the experimental task (i.e., after the binary choices). One advantage to this feature is that it allows for a consistency check within a single experimental session. The researcher can also define the desired weight ratio between the most and the least important attributes.

6 . When making choices in the real world, there are many situations in which an individual might prefer to forgo the alternatives in a given pair. For example, if both alternatives are very unattractive and/or very undifferentiated, the subject might simply wish to choose neither of them. In DSMAC, the researcher can include a neither option, allowing subjects simply to forgo both alternatives in a given pair.

7. Suppose that a given experimental task consists of making 5 binary choices between competing universities to attend. Furthermore, assume that the universities are defined by 10 attributes. A trial configuration table of size $5 \times 10$ is used to specify, for each of the 10 attributes defining the stimulus and for each of the 5 binary choices, the size of the attribute difference (i.e., small, medium, or large) and whom it favors. For example, denoting small, medium, and large attribute differences by $\mathrm{s}, \mathrm{m}$, and $\mathrm{l}$, respectively, a partial configuration for a given trial might look as follows: $-s,-1,+m,-m,+s$. The $+1-$ signs indicate which of the two alternatives is superior on an attribute, whereas the magnitude of attribute differences is specified by $\mathrm{s}, \mathrm{m}$, or 1 . Thus, cell $(i, j)$ of the table corresponds to the trial configuration value for binary choice $i$ and attribute $j$ (i.e., a subject's $j$ th most important attribute). Hence, in the above partial configuration, if the first and second pieces of information requested were a subject's second and fourth most important attributes, respectively, the corresponding attribute differences would be -1 and $-m$. Within DSMAC, the researcher interactively enters all of the trial configuration values (see Figure 1). 


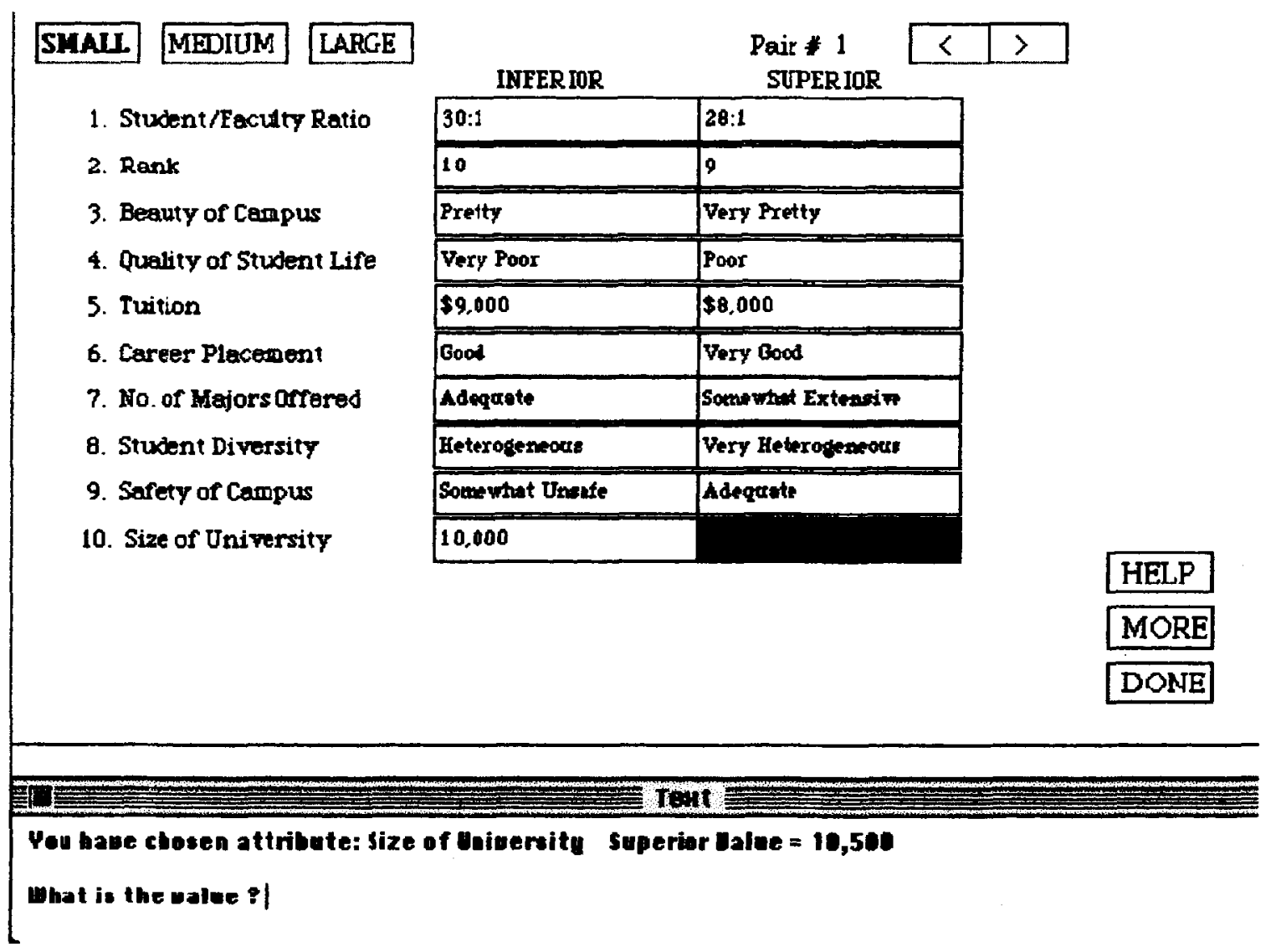

Figure 2. The experimenter enters pairs of attribute values corresponding to small, medium, and large differences for each of the attributes defining the stimulus in question. In cases in which an experiment has a large number of attributes defining the stimulus, these will not all fit on a single screen. Clicking on the More box allows one to view the next set of attributes. In the current experiment, there are 10 attributes defining the stimulus (i.e., they all fit on one screen) and 2 pairs of attribute values for a given magnitude of attribute difference. In other words, the experimenter would have to enter values onto six screens (3 magnitudes of attribute differences $\times 2$ pairs per magnitude).

8. The actual attribute values for a given attribute difference must also be specified. In other words, the attribute differences corresponding to small, medium, and large differences must be specified for each attribute. Referring to the university example, the experimenter might subjectively define a small attribute difference for tuition as anything under $\$ 1,500$, in which case pairs of attribute values might include $(\$ 5,000, \$ 5,400),(\$ 12,000, \$ 12,200)$, and $(\$ 8,000, \$ 9,000)$. All pairs of attribute values (for each of the three magnitudes of differences) are entered interactively by the researcher (see Figure 2).

In the above university example, there are three pairs of attribute values corresponding to a small difference on tuition. Setting the parameter to a particular value binds all attributes and their corresponding set of three magnitude differences to that number. In other words, if the researcher sets the parameter to five, each of the three magnitudes of attribute differences across all attributes will require that five pairs of attribute values be entered.

9. If the attribute selection screen consisted solely of a listing of the attributes, one might expect the acquisition order to be largely determined by the attributes' impor- tance weights (Aschenbrenner, Bockenholt, Albert, \& Schmalhofer, 1986; Saad, 1998a). Clearly, there are numerous other factors that might affect the order of attribute acquisitions. For example, an attribute's acquisition cost might also influence when it will be acquired (see Hagerty \& Aaker, 1984, for a discussion of other factors). In DSMAC, the researcher can specify up to three factors of acquisition order to be included in the attribute selection screen. For example, he or she might wish to include acquisition costs as a factor. Accordingly, the attributes would be listed with their corresponding acquisition costs. Hence, a subject would have to trade off the attributes' importance weights with their acquisition costs in determining which attribute to acquire next.

The researcher can choose from one of four predefined factors: acquisition costs, uncertainty about the acquisition costs, likelihood of obtaining information when it is requested, and the expected range/variance of attributes. Acquisition costs are specified through the use of time delays (in seconds), entered as any integer value greater than or equal to zero. Often in the real world, one cannot know the exact likelihood of incurring a specific acqui- 
DOAE

Student/Faculty Ratio

Rank

Beauty of Campus

Quality of Student Life

Tuition

Career Flacement

No. of Majors Orfered

stuatent Diversity

Safety of Campus

Size of University
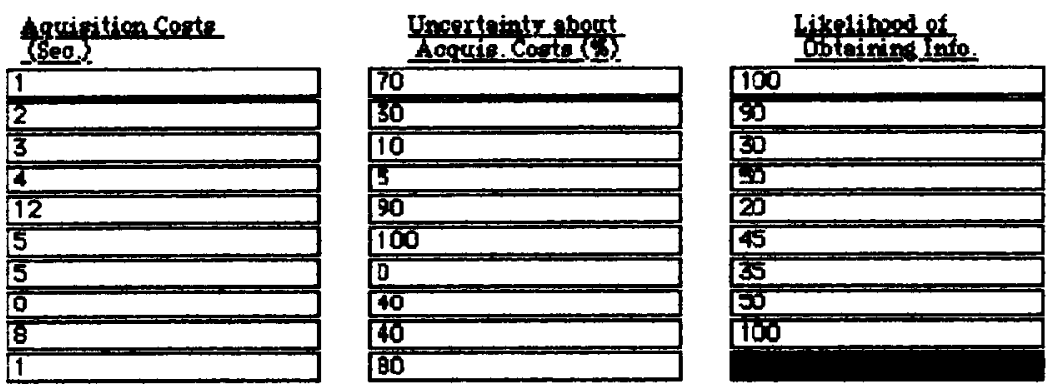

Tent sition cost. If a consumer needs to drive to a car dealership to acquire a specific piece of attribute information, its acquisition cost will depend on traffic flow that day. Accordingly, DSMAC permits the researcher to specify a stochastic component to each acquisition cost. For this second factor, the researcher can enter any integer value ranging from 0 to 100 . As such, a value of 70 implies that there is a $70 \%$ chance of not incurring any cost, with a corresponding $30 \%$ chance of incurring it (see Figure 3 ).

A third predefined factor is the likelihood of obtaining the information when it is requested. Referring to the above example, the individual in question might drive to the car dealership and find that it is closed. In other words, one might request to view information and not obtain it, while nonetheless incurring the corresponding acquisition cost. Here, too, the researcher enters any integer value ranging from 0 to 100 . A value of 80 means that there is an $80 \%$ chance of obtaining the information if it is requested, with a corresponding $20 \%$ of not obtaining it. Both the uncertainty about the acquisition costs and the likelihood of obtaining information when it is requested are implemented through the use of a random generator. If both factors are present within the same experiment, the interface uses a specific precedence order. A first random generator will determine whether the cost will be incurred, and a second one will determine whether the information will be shown when it is requested. If the former yields a value that implies that the cost is to be incurred, the time delay will be implemented, irrespective of the results of the second random generator. The default values for acquisition costs, uncertainty about the acquisition costs, and the likelihood of obtaining information when it is requested are set to 0,0 , and 100 , respectively.

The fourth and final predefined factor of acquisition order is the expected variance/range of an attribute. For example, if the competing alternatives are unlikely to be differentiated on a specific attribute, the expected value of acquiring this information would be small (Hagerty \& Aaker, 1984; Johnson \& Puto, 1987). Suppose that an individual is choosing between pairs of competing apartments. Rent information is likely to yield greater impact if rents vary between $\$ 200$ and $\$ 800$, as opposed to all being in the $\$ 400-\$ 500$ range. This factor only accepts semantic descriptors as possible values (e.g., very undifferentiated to very differentiated). The researcher can choose any descriptive scale to capture the range/variance values.

Finally, the researcher can specify up to three userdefined factors of acquisition order. For example, the ambiguity of attribute information and its accuracy are two such factors. Suppose that the experimental task involves making choices between two professors offering the same course. Information about the professors' overall personalities is likely to be more ambiguous than information about specific character traits (e.g., predisposition to be helpful), whereas departmental teaching ratings will likely be more accurate than students' wordof-mouth. The researcher can choose any descriptive scale to capture the values of the user-defined factors (e.g., very unambiguous to very ambiguous).

10. DSMAC contains a referential integrity check to ensure that all of the data structures are complete. Should empty cells be located, an error flag appears, with their exact locations specified. Thus, the subject module cannot be run if it uses an incomplete experiment as input.

\section{EDITING OF AN EXISTING EXPERIMENT}

DSMAC permits the researcher not only to create a new experiment but also to modify an existing one. The editing menu is intelligent in that, if a modification to one parameter requires corresponding alterations to other parameters, the interface will direct the researcher 
Modify Decisional Domain Labels.

Modify the Number of Atributes.

Modify Attribute Names / Directionality.

Modify Description of Attribute Scales.

Importance Weight Ratio.

Modify Attribute Re-Ranking Option.

Modify the Number of Binary Choices.

Modify the Neither Option.

Modify the Trial configuration Table.
Modify the Number of Pairs of Differences.

Modify the Pairs of Differences Values.

Modify the Factors of Acquisition Order.

Modify Online BackT racking Option.

Modify the Format for Attribute Listings.

Perform the Referential Integrity Check.

Save / Save as -- Experiment.

\section{OUIT}

Figure 4. This is the main menu for editing an existing experiment. The experimenter simply chooses the parameter that he or she wishes to modify.

through the various interrelated modifications. For example, assume that a current experiment has 10 binary choices and 15 attributes defining the stimulus. Accordingly, the trial configuration table would be a $10 \times 15$ array. Furthermore, suppose that the researcher wishes to increase the number of binary choices from 10 to 12 . DSMAC would immediately recognize that, as a result of adding 2 binary choices, the trial configuration table would now require two additional rows. See Figure 4 for the full editing menu.

\section{USE OF DSMAC IN EMPIRICAL RESEARCH}

Thus far, DSMAC has been used in two separate studies. Using apartment selection as the decisional domain, Saad (1998b) investigated the effects of dysphoria-a transient state of depression - on each of the three stages of the sequential choice process. Surprisingly, null effects were found at each stage. Saad and Boyle (1998) used two decisional domains - namely, choosing between pairs of bikes and pairs of cameras-to explore the effects of product expertise in a sequential choice task.

\section{CONCLUSION}

Although the subject module of DSMAC contains many substantial improvements over the original SMAC interface, DSMAC's key contribution lies in its experimenter module. One of the most time-consuming endeavors in experimental research is the long set-up time required for the running of new studies. The ability of a researcher to quickly alter the experimental task environment with the experimenter module of DSMAC fully alleviates this potential source of great frustration. For a minimal fee of $\$ 5.00$ (to cover postage and diskettes), in- terested researchers can obtain copies of the experimenter and subject modules of DSMAC and of the corresponding instructional documentation by writing directly to the author.

\section{REFERENCES}

Aschenbrenner, K. M., AlberT, D., \& Schmalhofer, F. (1984). Stochastic choice heuristics. Acta Psychologica, 56, 153-166.

Aschenbrenner, K. M., Bockenholt, U., Albert, D., \& SchmalHOFER, F. (1986). The selection of dimensions when choosing between multiattribute alternatives. In R. W. Scholz (Ed.), Current issues in West German decision research (pp. 63-78). Frankfurt: Lang,

Busemeyer, J. R., \& Townsend, J. T. (1993). Decision field theory: A dynamic-cognitive approach to decision making in an uncertain environment. Psychological Review, 100, 432-459.

DIEDERICH, A. (1995). A dynamic model for multi-attribute decision problems. In J.-P. Caverni, M. Bar-Hillel, F. H. Barron, \& H. Jungermann (Eds.), Contributions to decision making-I (pp. 175-191). Amsterdam: Elsevier, North-Holland

HAGERTY, M. R., \& AAKER, D. A. (1984). A normative model of consumer information processing. Marketing Science, 3, 227-246.

Johnson, M. D., \& PUTO, C. P. (1987). A review of consumer judgment and choice. In M. J. Houston (Ed.), Review of marketing (pp. 236292). Chicago: American Marketing Association.

SAAD, G. (1996). SMAC: An interface for investigating sequential multiattribute choices. Behavior Research Methods, Instruments, \& Computers, 28, 259-264.

SAAD, G. (1998a). Attribute acquisition and information integration in sequential choice. Manuscript submitted for publication.

SAAD, G. (1998b). Does dysphoria affect performance in a sequential multiattribute choice task? Manuscript submitted for publication.

SAAD, G., \& BOYLE, P. (1998, July). When do experts close up shop: $A$ study of the use of sequential information in a purchase decision. Paper to be presented at the Marketing Science Conference, Fontainebleau, France.

SAAD, G., \& Russo, J. E. (1996). Stopping criteria in sequential choice. Organizational Behavior \& Human Decision Processes, 67, 258-270.

WALD, A. (1947). Sequential analysis. New York: Wiley.

(Manuscript received October 15, 1997; revision accepted for publication February 19,1998 .) 